\title{
Eine neue Form von Glas durch molekulares Auffädeln
}

\author{
Physiker der Universität Wien haben in Zusammenarbeit mit dem Max-Planck-Institut für \\ Polymerforschung in Mainz eine neue Art von Glas entdeckt, das sich aus langen ringförmigen \\ Molekülen bildet. Indem die Wissenschaftler Teile der Ringe beweglicher machen, verwickeln \\ sich die Ringe stärker ineinander und die molekulare Flüssigkeit bildet ein Glas.
}

Materialien aus Glas sind im täglichen Leben allgegenwärtig und reichen von Fensterscheiben über PET-Flaschen bis hin zu Espressotassen aus Porzellan. Glas entsteht durch rasches Abkühlen von geschmolzenen Teilchen, wie beispielsweise Siliziumdioxid bei Fenstern oder Polymeren in Kunststoffflaschen. Die Eigenschaften solcher Stoffe wie Transparenz oder Zugfestigkeit ergeben sich aus ihrer mikroskopisch ungeordneten und dichten Struktur. In heißem Zustand können sich alle Teilchen frei aneinander vorbei bewegen und so beispielsweise beim Glasblasen neu angeordnet werden. Beim Abkühlen der Schmelze wird jedoch jedes Teilchen von seinen Nachbarn eingeengt, was das Glas fest und formbeständig macht.

Vor etwa 25 Jahren stellten Wissenschaftler die Vermutung auf, dass es eine weitere, neue Art von Glas geben könnte. Die Bestandteile dieses neuartigen Glases sind keine linearen Molekülketten mehr, sondern sehr lange Ringpolymere. Solche „Ringe“ können sich gegenseitig auffädeln, das heißt ein Ring fädelt sich durch das Auge eines anderen Rings. Dabei schränken sich die Ringe in ihrer Bewegung gegenseitig ein. Wenn viele Ringe aufge- fädelt sind, müssen sich mehrere Ringe nach und nach voneinander lösen, damit auch nur ein einziges Makromolekül freigesetzt wird. Solange ein derartiger Prozess bloß durch thermische Fluktuationen gesteuert wird, benötigt der Ring lange Zeit um sich zu befreien und die vorhergesagte ungeordnete Struktur verhält sich de facto wie ein festes Glas (Bild 1).

Ein solch glasartiger Zustand wurde jedoch noch in keinem Experiment beobachtet. Dies ist möglicherweise auf die derzeit zu kurz synthetisierbaren Ringpolymere zurückzuführen. Für derzeit verfügbare Ringlängen zeigten die vorherigen Computersimulationen, dass solches Glas nur durch schwer zu erreichende Bedingungen wie beispielsweise künstliches Einfrieren der Ringe herstellbar wäre. Es schien, als ob ein echtes „topologisches Glas", ein aus stark verwickelten ringförmigen Molekülen bestehendes Glas, weiterhin unerreichbar bliebe.

„Wir haben den zu bisherigen Versuchen umgekehrten Weg eingeschlagen, ein topologisches Glas zu finden. Anstelle des unphysikalischen Einfrierens der Ringe haben wir in unseren Simulationen einige Ringsegmente beweglicher gemacht. Das gelingt uns, indem

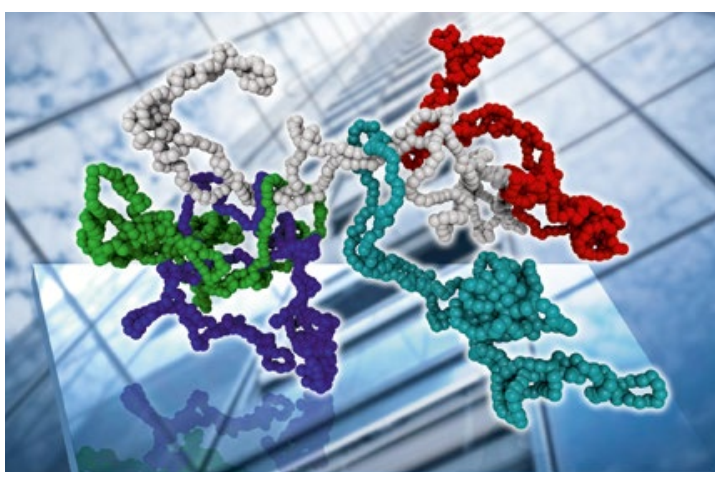

Bild 1 Ringmoleküle (differenziert nach Farben) weisen gegenseitige Einfädelungen auf, die ihre Bewegung hemmen und die Ausbildung des Glases ermöglichen. (๑ Uni Wien / MPI-P) wir Teile der Ringe zu stärkeren Fluktuationen zwingen“, erklären Iurii Chubak und Jan Smrek, Kollegen an der Universität Wien und gemeinsame Hauptautoren der Studie. „Diese stärkeren Fluktuationen können durch das Einbetten von molekularen Motoren - Molekülen mit lokal auf Kosten der Energie ausgeübten Kräften - verwirklicht werden. Eine weitere Möglichkeit ist die Synthese von Ringen, die Segmente mit erhöhter Lichtabsorption enthalten. Solche aktiv angetriebenen Ringe fädeln und verwickeln sich dann so stark, dass sie sich praktisch nicht aneinander vorbeibewegen können. Bemerkenswert ist, dass wir das topologische Glas bei experimentell zugänglichen Ringlängen und Antriebskräften beobachten“, so Jan Smrek, der seine Arbeit in Zusammenarbeit mit dem MaxPlanck-Institut für Polymerforschung in Mainz und unter Förderung durch das LiseMeitner-Programm des Wissenschaftsfonds FWF durchgeführt hat.

„Dieses Glas unterscheidet sich mikroskopisch stark vom Material der Flasche, aus der Sie normalerweise Ihr Lieblingsgetränk trinken. Detailliertere Materialeigenschaften des aktiven topologischen Glases werden in Zukunft weiter untersucht werden. Aber schon jetzt ist es spannend, nicht nur aus Sicht der Grundlagenforschung, sondern auch aufgrund der möglichen Anwendungen, beispielsweise flüssiges Material mit umkehrbarer Verglasung bei Lichteinwirkung", ergänzt Seniorautor Christos Likos von der Fakultät für Physik der Universität Wien.

Kontakt:

MPI für Polymerforschung, Mainz, www.mpip-mainz.mpg.de 